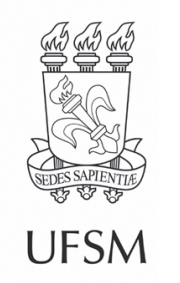

\title{
Artigos
}

\section{Efeito da temperatura no desenvolvimento e na reprodução de Blastopsylla occidentalis (Hemiptera: Aphalaridae) em condições de laboratório}

\author{
Effect of temperature on the development and reproduction of Blastopsylla \\ occidentalis (Hemiptera: Aphalaridae) under laboratory conditions
Talita Benedcta Santos Künast ${ }^{\odot}$, Dalva Luiz de Queiroz ${ }^{\odot}{ }^{\circ}$, Daniel Burckhardt ${ }^{\prime \prime \prime}{ }^{\circledR}$, Ednaldo Antônio de Andrade ${ }^{\circledR}$, Marliton Rocha Barreto ${ }^{10}$ \\ ' Universidade Federal de Mato Grosso, Sinop, MT, Brasil \\ "Embrapa Florestas, Colombo, PR, Brasil \\ II' Naturhistorisches Museum Basel, Basel, Switzerland
}

\section{RESUMO}

Blastopsylla occidentalis Taylor,1985 (Hemiptera: Aphalaridae) é um psilídeo que tem como hospedeiro o eucalipto, originário da Austrália, foi introduzido no Brasil em meados da década de 1990. Devido à escassez de informações a respeito da biologia desse inseto, este trabalho teve como objetivo conhecer o efeito da temperatura no desenvolvimento e reprodução do psilídeo sob condições de laboratório. 0 experimento foi realizado entre 2018 e 2019, em câmaras climatizadas reguladas em cinco temperaturas $\left(15,20,25,30\right.$ e $\left.35^{\circ} \mathrm{C}\right)$. Os parâmetros observados nessa etapa do experimento foram: período de desenvolvimento dos ovos (número de dias, da postura até a eclosão); taxa de eclosão de imaturos; desenvolvimento de imaturos; taxa de emergência de adultos; longevidade, o período (em dia) de postura até a morte dos adultos da primeira geração $\left(F_{1}\right)$. Como resultado obteve-se o ciclo completo nas temperaturas 20,25 e $30^{\circ} \mathrm{C}$, com duração média de $32,78,27,76$ e 28,44 dias, respectivamente. Para a geração $F_{1}$, não foram observadas postura na temperatura $30^{\circ} \mathrm{C}$, enquanto em 20 e $25^{\circ} \mathrm{C}$, a fecundidade média das fêmeas foi de 19,04 e 26,40, respectivamente. A duração média do período de incubação de ovos foi de $6,70\left(20^{\circ} \mathrm{C}\right)$ e 5,59 dias $\left(25^{\circ} \mathrm{C}\right)$. Portanto, infere-se que as temperaturas 15 e $35^{\circ} \mathrm{C}$ são limitantes para o desenvolvimento do inseto, pois, durante o estudo, os imaturos de Blastopsylla occidentalis não completaram o ciclo de desenvolvimento. A faixa de temperatura ótima para o desenvolvimento e reprodução foi entre 20 e $25^{\circ} \mathrm{C}$, sendo que em $25^{\circ} \mathrm{C}$ foi a temperatura em que o inseto apresentou menor mortalidade em todas as fases, menor tempo de desenvolvimento e maior número médio de ovos/dias. Espera-se que esses resultados sirvam para conduzir estudos para a aplicação de técnicas de manejo para eventual controle da espécie.

Palavra-chave: Eucalipto; Exigências térmicas; Proteção florestal; Psylloidea 


\begin{abstract}
Blastopsylla occidentalis Taylor, 1985 (Hemiptera: Aphalaridae) is a psyllid which host is the eucalyptus, originally from Australia, which was introduced in Brazil in the mid-1990s. Little is known on the biology of this insect. Here, we examine the effect of temperature on its development and the reproduction under laboratory conditions. The experiments were carried out in 2018 and 2019, in climate chambers regulated at five temperatures $\left(15,20,25,30\right.$ and $\left.35^{\circ} \mathrm{C}\right)$. The parameters observed at this stage of the experiment were: period of the egg development (number of days, from laying to hatching); hatching rate of immatures; development of immatures; adult emergency rate; longevity, the period (in day) of laying until the death of the first generation adults (F1). Blastopsylla occidentalis completed the life cycle at temperatures of 20,25 and 30C, with an average duration of 32.78, 27.76 and 28.44 days, respectively. In the $\mathrm{F} 1$ generation, no posture was observed at $30^{\circ} \mathrm{C}$, while at 20 and $25^{\circ} \mathrm{C}$, the average fertility was 19.04 and 26.40 eggs per female, respectively. The average duration of the egg incubation was 6.70 (20 $\left.{ }^{\circ} \mathrm{C}\right)$ and $5.59\left(25^{\circ} \mathrm{C}\right)$ days. Immature B. occidentalis did not complete their development at 15 and $35^{\circ} \mathrm{C}$ and we conclude that these temperatures limit its development. The optimum temperature range for the development and the reproduction was between 20 and $25^{\circ} \mathrm{C}$, with $25^{\circ} \mathrm{C}$, being the temperature at which the insect had the lowest mortality in all stages, the shortest development time and the highest average number of eggs/days. It is hoped that these results will help to improve the management techniques for the control of the species.
\end{abstract}

Keyword: Eucalypt; Thermal requirements; Forest protection; Psylloidea

\title{
1 INTRODUÇÃO
}

A representatividade do Brasil no mercado internacional de produtos florestais cresce a cada dia. Segundo a Indústria Brasileira de Árvores (IBÁ), o Brasil é o segundo maior produtor de celulose no mundo, com uma extensão de 7,83 milhões de hectares de floresta plantada e com, aproximadamente, 5,7 milhões de hectares pertence ao cultivo de eucalipto (INDÚSTRIA BRASILEIRA DE ÁRVORES, 2019). Nativo da Austrália e pertencente à família das mirtáceas, o eucalipto está se destacando no Brasil pela adaptabilidade ao clima tropical, o que permite um crescimento contínuo associado ao rápido acúmulo de biomassa (LONGUE-JÚNIOR; COLODETTE, 2013).

Com a crescente área plantada de eucalipto no país, o relato de pragas exóticas tem aumentado consideravelmente nas últimas décadas (SCHÜHLI et al., 2016). Entre as pragas introduzidas em meados da década de 1990 e início dos anos 2000, os insetos da superfamília Psylloidea se destacam, pois são encontrados amplamente 
distribuídos nos plantios de eucalipto, causando danos (BURCKHARDT; OUVRARD, 2012; QUEIROZ et al., 2018).

No Brasil, foram introduzidas quatro espécies de psilídeos da família Aphalaridae: Ctenarytaina eucalypti Maskell, 1890; Ctenarytaina spatulata Taylor, 1997; Blastopsylla occidentalis Taylor, 1985 e Glycaspis brimbecombei Moore, 1964 e a primeira coleta de Blastopsylla occidentalis no país ocorreu em 1997, em plantas de Eucalyptus urophylla e híbridos de Eucalyptus urophylla com Eucalyptus grandis (QUEIROZ et al., 2018).

Os adultos de Blastopsylla occidentalis são insetos pequenos que medem entre 1,5 a 2,0 mm, suas asas possuem veias marrons com a presença de uma membrana cinza, a coloração amarelada é predominante na espécie, com diferenças na tonalidade, em que machos são geralmente amarelos e as fêmeas são mais escuras (BURCKHARDT; ELGUETA, 2000). Como outros psilídeos, os imaturos e adultos de Blastopsylla occidentalis se alimentam da seiva do floema.

Em viveiros comerciais pode acarretar prejuízos econômicos, uma vez que tem preferência por brotações e folhas mais jovens, o que pode afetar o crescimento das mudas comprometendo a produtividade (QUEIROZ et al., 2017). Em plantios já estabelecidos, com plantas adultas, o inseto concentra-se principalmente nas ponteiras apicais, ocasionando o atrofiamento das plantas, distorção e manchas em folhas e pecíolos, contribuindo para a perda de vigor em toda a extensão da planta atacada (SANTANA; BURCKHARDT, 2007; QUEIROZ; BURCKHARDT; MAJER, 2012). Quanto aos sintomas de ataque, os danos mais rigorosos são evidenciados quando há uma quantidade elevada de imaturos e adultos nos ápices e folhas mais jovens, independentemente da fase em que a planta se encontra (QUEIROZ et al., 2017).

A presença de Blastopsylla occidentalis já é relatada em vários estados brasileiros, principalmente nos que possuem plantios comerciais em grande escala e em regiões com deficit hídrico acentuado em alguma época do ano (QUEIROZ; BURCKHARDT; MAJER, 2012; SALIBA et al., 2019). Vários autores mencionam que além da disponibilidade de alimento, a temperatura também poderá afetar a bioecologia de psilídeos, assim 
como de outros insetos da ordem Hemiptera, desse modo, poderá influenciar no tempo de desenvolvimento e como será sua dinâmica populacional (OLIVEIRA et al., 2010; QUEIROZ et al., 2010; FONSECA et al., 2016).

Problemas fitossanitários nos plantios de eucalipto são motivos de grande preocupação para o setor florestal, e para solucionar esses problemas, é necessária a adoção de medidas que poderão elevar os custos de produção, afetando negativamente a lucratividade do produto final (VAN LIEROP et al., 2015). Estima-se que uma extensão superior a um milhão de hectares de florestas plantadas na América do Sul foram atacadas por insetos-praga (FOOD AND AGRICULTURE ORGANIZATION OF THE UNITED NATIONS, 2015).

Diferente dos outros insetos-praga associados ao eucalipto, Blastopsylla occidentalis possui escassez de informações relacionadas a sua biologia. Sendo este o primeiro estudo dessa natureza, a fim de preencher essa demanda. Portanto, o objetivo deste foi conhecer o efeito da temperatura no desenvolvimento e reprodução de Blastopsylla occidentalis em condições de laboratório.

\section{MATERIAL E MÉTODOS}

\section{1 Área de estudo}

A pesquisa foi realizada no Laboratório de Entomologia do Acervo Biológico da Amazônia Meridional - ABAM da Universidade Federal de Mato Grosso, campus Sinop (11 $\left.{ }^{\circ} 51^{\prime} 51.4^{\prime \prime} \mathrm{S} 55^{\circ} 28^{\prime} 58.4^{\prime \prime} \mathrm{O}\right)$. Para realização dos experimentos, casais de Blastopsylla occidentalis foram coletados em eucaliptos plantados na Fazenda Cunhatay $\left(11^{\circ} 45^{\prime} 19,4^{\prime \prime}\right.$ S; 55²3'14,1" O), localizada no município de Sinop, Mato Grosso, e, também, de insetos da criação realizada em casa de vegetação. Todos os insetos foram mantidos em temperatura ambiente até o início dos experimentos. 


\subsection{Manejo e definição do clone de eucalipto para estudo biológico}

Mudas clonais de eucalipto, com aproximadamente 90 dias de idade, de híbridos Eucalyptus grandis $x$ Eucalyptus urophylla $(\mathrm{H}-13)$; Eucalyptus urophylla $\times$ Eucalyptus grandis (I-144); Eucalyptus urophylla x Eucalyptus camaldulensis (VM-01), foram plantadas em copos descartáveis de $180 \mathrm{ml}$. Quinzenalmente, receberam adubação com solução nutritiva (QUEIROZ et al., 2020) e foram isoladas por 40 dias para fixação do plantio, até serem infestadas em gaiolas teladas medindo $80 \times 80 \times 80 \mathrm{~cm}$.

Para selecionar o clone foram realizadas cinco infestações, sendo que, em cada uma, quinze casais de Blastopsylla occidentalis foram isolados em três gaiolas que possuíam dez mudas de cada clone, pelo período de 24 horas para o acasalamento e postura das fêmeas. Após esse período, os casais foram retirados com auxílio do aspirador entomológico e liberados em casa de vegetação, para a manutenção de criação do inseto. Dentre os três clones avaliados, o VM-01 foi selecionado, considerando que neste clone foi observado o maior número de posturas.

\subsection{Estudo biológico no laboratório}

Para acompanhar o ciclo de desenvolvimento foram realizadas quatorze infestações ( 1 infestação = 30 mudas isoladas). Após a realização da infestação, as mudas foram avaliadas, e quantificados o número de ovos presentes, em seguida estas ficaram isoladas individualmente em gaiolas cilíndricas confeccionadas em polipropileno transparente medindo $30 \times 20 \mathrm{~cm}$ com abertura na parte inferior, a qual foi acoplada no copo que continha a muda, e na superior foi fixada tela tipo "voile" para ventilação (Figura 1), e alocadas em câmaras climatizadas tipo Biochemical Oxigen Demand (BOD), reguladas em temperaturas de $15,20,25,30$ e $35^{\circ} \mathrm{C}$, fotoperíodo de $12 / 12$ horas e umidade relativa de $70 \%$.

Os parâmetros observados, diariamente, nessa etapa do experimento foram: incubação: período de desenvolvimento dos ovos, ou seja, número de dias, desde a postura até a eclosão dos imaturos; taxa de eclosão de imaturos: porcentagem 
[(número de imaturos) /(número de ovos) x 100]; desenvolvimento de imaturos: período (em dia), desde a eclosão até a emergência do adulto; percentual de mortalidade de imaturos que não chegaram à fase adulta; taxa de emergência de adultos: porcentagem [(número de adultos) / (número de imaturos) x 100]; longevidade: número de dias de sobrevivência na fase adulta e ciclo completo: período (em dia) de postura até a morte dos adultos.

Figura 1 - Experimento com Blastopsylla occidentalis Taylor, 1985 (Hemiptera: Aphalaridae); A: modelo de gaiolas utilizadas; B: Mudas de eucalipto infestadas com ovos do inseto

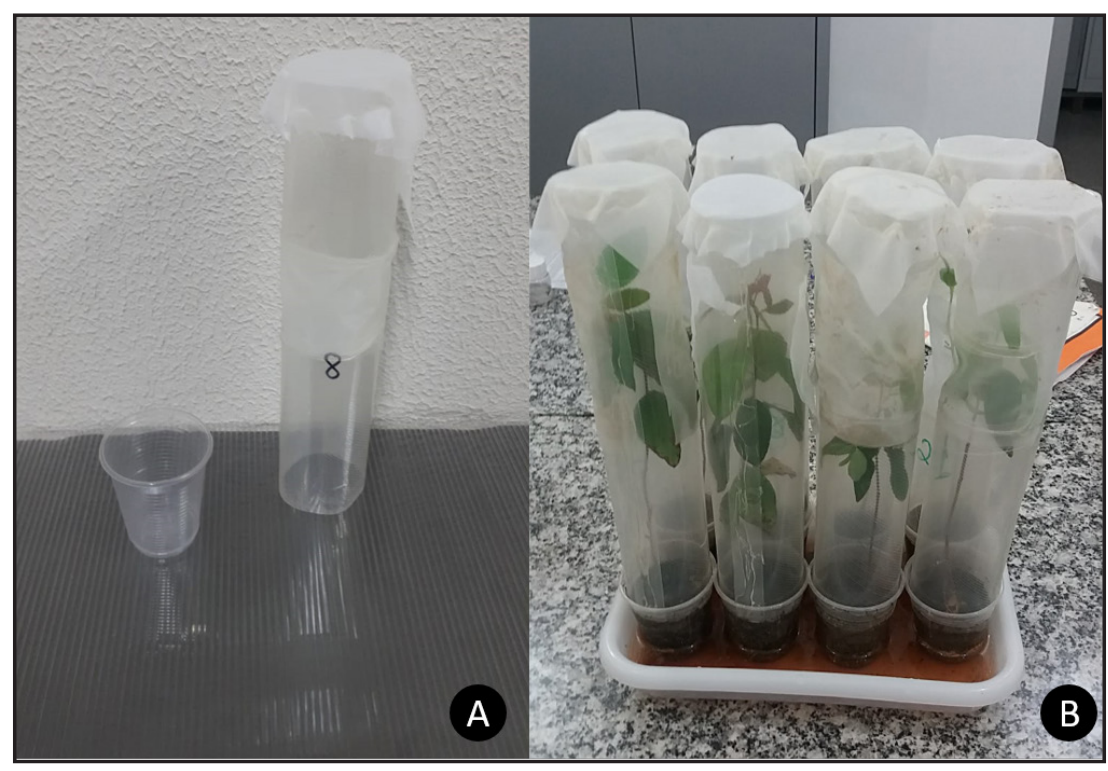

Fonte: Autores (2020)

Para obtenção da geração $F_{1}$ esperou-se de 24 a 48 horas após a emergência dos adultos para realizar a remoção dos insetos das plantas com o aspirador entomológico, e com auxílio do microscópio estereoscópico foi realizada a sexagem e separação dos casais. Em uma nova gaiola, com mudas de eucalipto livre de infestação, ocorreu a liberação dos casais, em suas respectivas temperaturas, os quais diariamente foram observados, até o surgimento da primeira postura e avaliação do parâmetro reprodutivo (quantidade de ovos/dia por fêmea), taxa de eclosão e duração do período de incubação de imaturos $F_{1}$. 


\subsection{Análise estatística}

Para todas as temperaturas estudadas, foi considerado repetição cada ovo observado de Blastopsylla occidentalis, sendo assim o número de repetições foi diferente entre as temperaturas. Os dados obtidos em cada parâmetro observado (período de incubação de ovos, desenvolvimento de imaturos, longevidade de adultos, ciclo total e período de incubação da geração $F_{1}$ ), foram submetidos a testes de normalidade e homocedasticidade e após a verificação desses pressupostos foi realizada a análise de variância (ANOVA) e as médias comparadas pelo teste de Scott-Knott a 5\% de probabilidade, com auxílio do programa estatístico Sisvar (FERREIRA, 2011).

\section{RESULTADOS E DISCUSSÃO}

Os resultados indicaram que as temperaturas de 20 e $25^{\circ} \mathrm{C}$ foram adequadas ao desenvolvimento do psilídeo, enquanto as temperaturas de 15 e $35^{\circ} \mathrm{C}$ foram limitantes, pois não houve emergência de adultos (Tabela 1). Laws e Belovsky (2010) mencionaram que os processos fisiológicos, comportamentais e de desenvolvimento dos insetos são afetados diretamente pela temperatura, uma vez que possuem capacidade limitada de termorregulação.

Hodkinson (2009) cita que as temperaturas quando não estão na faixa ideal para o desenvolvimento dos psilídeos, pode resultar em desenvolvimento mais lento e alta taxa de mortalidade. Fato que se observa na temperatura mais baixa deste estudo $\left(15^{\circ} \mathrm{C}\right)$, em que a duração média do período incubação foi de 18,30 $\pm 0,16$ dias, sendo esse o período mais prolongado para eclosão de imaturos e limitante para o desenvolvimento de Blastopsylla occidentalis. Wilcken et al.(2015) observaram que a temperatura de $18^{\circ} \mathrm{C}$ é limitante para o desenvolvimento de Glycaspis brimblecombei, sendo assim superior ao encontrado para Blastopsylla occidentalis.

A taxa de eclosão de imaturos em $15^{\circ} \mathrm{C}$ foi de $35 \%$ (Tabela 1 ), sendo limitante para o desenvolvimento do inseto. Santa-Cecília et al. (2011) relataram que a temperatura 
foi um fator limitante em seus estudos e que a mortalidade elevada em $15^{\circ} \mathrm{C}$ pode estar atribuída à redução no metabolismo dos indivíduos, o que resulta em maior tempo para desenvolvimento nos estágios de imaturos. Queiroz et al. (2020) citam que em condições de casa de vegetação, em Colombo - Paraná, o psilídeo Blastopsylla occidentalis não tolera temperaturas muito baixas.

Tabela 1 - Número de ovos, média \pm erro padrão e intervalo de variação do período incubação e taxa de eclosão, número e taxa mortalidade de imaturos, total e taxa de emergência de adultos de Blastopsylla occidentalis Taylor, 1985 (Hemiptera: Aphalaridae) em cinco temperaturas em BOD. Sinop - MT, Brasil

\begin{tabular}{|c|c|c|c|c|c|c|c|c|}
\hline \multirow[b]{2}{*}{$\begin{array}{c}\text { Temperatura } \\
\left({ }^{\circ} \mathrm{C}\right)\end{array}$} & \multirow[b]{2}{*}{ Ovos } & \multicolumn{3}{|c|}{ Incubação } & \multicolumn{2}{|c|}{ Imaturos } & \multicolumn{2}{|c|}{ Adultos } \\
\hline & & $\begin{array}{c}\text { Média } \pm \\
\text { EP } \\
\text { (dias) }\end{array}$ & $\begin{array}{c}\text { Intervalo } \\
\text { de } \\
\text { variação }\end{array}$ & $\begin{array}{l}\text { Eclosão } \\
\text { (\%) }\end{array}$ & Número & $\begin{array}{c}\text { Mortalidade } \\
\text { (\%) }\end{array}$ & Número & $\begin{array}{c}\text { Emergência } \\
(\%)\end{array}$ \\
\hline 15 & 259 & $\begin{array}{c}18,30 \pm \\
0,16 d\end{array}$ & $14-20$ & 35 & 90 & 100 & 0 & 0 \\
\hline 20 & 362 & $\begin{array}{l}7,42 \pm \\
0,06 b\end{array}$ & $6-9$ & 67 & 243 & 53 & 114 & 47 \\
\hline 25 & 400 & $\begin{array}{l}5,95 \pm \\
0,04 a\end{array}$ & $5-8$ & 91 & 362 & 34 & 238 & 65 \\
\hline 30 & 420 & $\begin{array}{l}8,03 \pm \\
0,05 c\end{array}$ & $6-11$ & 79 & 331 & 69 & 101 & 31 \\
\hline 35 & 236 & $\begin{array}{l}7,49 \pm \\
0,16 b\end{array}$ & $6-10$ & 25 & 59 & 100 & 0 & 0 \\
\hline
\end{tabular}

Fonte: Autores (2020)

Em que: Médias seguidas pela mesma letra na coluna, não diferem significativamente entre si pelo teste de Scott-Knott a 5\% de probabilidade.

$\mathrm{Na}$ temperatura mais alta do estudo $\left(35^{\circ} \mathrm{C}\right)$ obteve-se a menor taxa de eclosão de imaturos (25\%) e o período incubação teve duração média de 7,49 (Tabela 1). Schowalter (2016) cita que os insetos quando expostos à temperatura acima do ideal elevam o custo metabólico acarretando em baixa nutrição e reduzindo a sobrevivência dos indivíduos. Colônias de Blastopsylla occidentalis se mantiveram em pleno desenvolvimento em condições de casa de vegetação, em que as temperaturas máximas chegaram aos $50^{\circ} \mathrm{C}$ (QUEIROZ et al., 2020). Vale ressaltar que nas condições 
deste experimento as temperaturas foram constantes durante todo o período e, nos trabalhos de Queiroz et al. (2020), as temperaturas flutuaram entre os dias e noites, sendo que as máximas e mínimas não perduraram por muitas horas.

Portanto, infere-se que os resultados aqui apresentados para o inseto em condições de laboratório são diferentes do observado em casa de vegetação, na qual a amplitude térmica oscila entre os períodos diurno e noturno, podendo assim favorecer o desenvolvimento não só desse psilídeo, mas também de outros insetos (SANTA-CECÍLIA et al.,2011; COLINET et al., 2015).

Analisando os resultados do período de incubação, as temperaturas $20^{\circ} \mathrm{C} \mathrm{e} 35^{\circ} \mathrm{C}$ apresentaram média próxima (Tabela 1), portanto não se diferiram estatisticamente, enquanto as demais temperaturas diferiram entre si, pela análise de médias do teste Scott-Knott. Em $20^{\circ} \mathrm{C}$, a média de duração do período de incubação foi de 7,42 , apresentando a taxa de eclosão de 67\%, com obtenção de 243 imaturos.

A temperatura de $25^{\circ} \mathrm{C}$ foi a que apresentou menor tempo para eclosão dos imaturos (5,95 dias). Enquanto, para $30^{\circ} \mathrm{C}$, a média foi de 8,03 \pm 0,05 dias (médias referentes a todos os imaturos que eclodiram, inclusive os que não se desenvolveram até a fase adulta). Corroborando os resultados do presente estudo, Rodrigues (2004) menciona que temperaturas próximas a $25^{\circ} \mathrm{C}$ estão dentro do limiar ótimo para o desenvolvimento da grande maioria dos insetos, visto que são nessas condições de temperatura o desenvolvimento é mais satisfatório.

Os indivíduos que completaram o ciclo de desenvolvimento em 20, 25 e $30^{\circ} \mathrm{C}$ apresentaram as médias do período de incubação de 6,97, 5,80 e 7,40 dias, respectivamente. A menor duração do período de desenvolvimento de imaturos foi em $25^{\circ} \mathrm{C}$ com 13 dias, e máxima de 26 dias a $20^{\circ} \mathrm{C}$ (Tabela 2). O ciclo completo de Blastopsylla occidentalis em $30^{\circ} \mathrm{C}$ foi de 28,44 dias, com um total de 101 adultos emergidos. Segundo Souza e Kirst (2010), insetos imaturos podem ter seu desenvolvimento reduzido ou acelerado dependendo da condição de temperatura ao qual esteja submetido e os resultados aqui apresentados corroboram essa afirmação. 
O desenvolvimento de imaturos foi de 21,5 dias $\left(20^{\circ} \mathrm{C}\right)$, sendo essa a maior duração média do período. Para o ciclo total de Blastopsylla occidentalis nessa temperatura a média foi de 32,78 dias, valores esses inferiores aos encontrados para Ctenarytaina spatulata, que apresentou média de 44,89 dias (ciclo total) (QUEIROZ; ZANOL, 2006). Fonseca et al. (2016) relataram que o efeito da temperatura pode variar entre as espécies e ressaltaram que as temperaturas mais baixas geralmente influenciam na duração do desenvolvimento biológico.

Tabela 2 - Número de indivíduos observados (N), duração média \pm erro padrão dos períodos de incubação e de desenvolvimento de imaturo, longevidade de adultos e taxa de mortalidade após 24 horas da emergência de adultos, ciclo total e seus respectivos intervalos de variação (dias) para o desenvolvimento biológico de Blastopsylla occidentalis Taylor, 1985 (Hemiptera: Aphalaridae), em cinco temperaturas. Sinop - MT, Brasil

\begin{tabular}{|c|c|c|c|c|c|c|}
\hline $\begin{array}{c}\text { Temperatura } \\
\left({ }^{\circ} \mathrm{C}\right)\end{array}$ & $\mathbf{N}$ & $\begin{array}{l}\text { Incubação } \\
\text { (dias) }\end{array}$ & $\begin{array}{l}\text { Desenvolvimento } \\
\text { imaturos (dias) }\end{array}$ & Longevidade & $\begin{array}{l}\text { Ciclo } \\
\text { total }\end{array}$ & $\begin{array}{c}\text { Mortalidade } \\
\text { em 24h após } \\
\text { a emergência } \\
\text { (\%) }\end{array}$ \\
\hline 20 & 114 & $\begin{array}{c}6,97 \pm 0,06 \\
b \\
(6-8)\end{array}$ & $\begin{array}{c}21,57 \pm 0,16 c \\
(17-26)\end{array}$ & $\begin{array}{c}4,22 \pm 0,15 b \\
(1-6)\end{array}$ & $\begin{array}{c}32,78 \pm \\
0,21 \mathrm{c} \\
(24-37)\end{array}$ & 13,15 \\
\hline 25 & 238 & $\begin{array}{c}5,80 \pm 0,11 \\
a \\
(5-8)\end{array}$ & $\begin{array}{c}15,77 \pm 0,11 a \\
(13-22)\end{array}$ & $\begin{array}{c}6,08 \pm 0,09 c \\
(1-8)\end{array}$ & $\begin{array}{c}27,76 \pm \\
0,10 a \\
(24-32)\end{array}$ & 0,84 \\
\hline 30 & 101 & $\begin{array}{c}7,40 \pm 0,07 \\
C \\
(6-8)\end{array}$ & $\begin{array}{c}17,93 \pm 0,18 b \\
(14-23)\end{array}$ & $\begin{array}{c}3,03 \pm 0,09 a \\
(1-4)\end{array}$ & $\begin{array}{c}28,44 \pm \\
0,18 b \\
(23-33)\end{array}$ & 9,90 \\
\hline
\end{tabular}

Fonte: Autores (2020)

Em que: Médias seguidas pela mesma letra na coluna, não diferem significativamente entre si pelo teste de Scott-Knott a 5\% de probabilidade.

Observou-se que a melhor temperatura para a incubação dos ovos e desenvolvimento de imaturos Blastopsylla occidentalis foi de $25^{\circ} \mathrm{C}$. Desse modo, os imaturos concluíram seu desenvolvimento em menor tempo (5,80 e 15,77 dias), com 
média de ciclo completo de 27,7 dias. Apresentando ainda a longevidade de adultos prolongada em comparação a 20 e $30^{\circ} \mathrm{C}$ (Tabela 2). Corroborando as informações, Hodkinson (2009) cita que o desenvolvimento da maioria das espécies de psilídeo está diretamente ligado à temperatura, e para se ter um desenvolvimento bem sucedido também é necessário que haja um sincronismo entre esses indivíduos e a planta hospedeira. Rodrigues (2004) menciona que em temperaturas próximas a $25^{\circ} \mathrm{C}$ os insetos têm o desenvolvimento mais rápido, o que ficou evidenciado nos dados biológicos aqui apresentados.

A análise estatística realizada para os dados biológicos resultou em diferença significativa em todos os parâmetros observados (Tabela 2). Para as três temperaturas, que foram observados o ciclo completo, a mortalidade de adultos nas primeiras 24 horas de emergência foi relativamente baixa, com taxas de: $13,15 \%\left(20^{\circ} \mathrm{C}\right) ; 0,84 \%$ $\left(25^{\circ} \mathrm{C}\right)$ e $9,90 \%\left(30^{\circ} \mathrm{C}\right)$. Confirmando, assim, a temperatura de $25^{\circ} \mathrm{C}$ como a ideal para a criação do inseto em condições de laboratório.

Nas temperaturas 20,25 e $30^{\circ} \mathrm{C}$ foi observada a capacidade reprodutiva de 38, 66 e 32 fêmeas, respectivamente (Tabela 3). Em $30^{\circ} \mathrm{C}$ houve desenvolvimento do inseto, porém, nessa temperatura, não foram observadas posturas, esse feito também foi observado para Glycaspis brimblecombei (FIRMINO, 2004). Em $30^{\circ} \mathrm{C}$ a longevidade do adulto foi reduzida e esse dado corrobora o trabalho de Barbosa et al. (2019), no qual relataram que temperaturas acima de $30^{\circ} \mathrm{C}$ podem contribuir para a redução de fecundidade e de sobrevivência. Ao estudarem Diaphorina citri Kuwayama, 1908 (Hemiptera: Liviidae) Hall, Wenninger e Hentz (2011) observaram que os limiares inferior e superior estimados para oviposição são de $16,0^{\circ} \mathrm{C}$ e $41,6^{\circ} \mathrm{C}$, respectivamente.

A temperatura exerceu influência na geração $F_{1}$ de Blastopsylla occidentalis, em consequência, as fêmeas provenientes das temperaturas 20 e $25^{\circ} \mathrm{C}$ tiveram as médias diária de 19,04 e 26,40 ovos, respectivamente. Sendo que a $25^{\circ} \mathrm{C}$, as fêmeas ovipositaram até 38 ovos por dia, enquanto em $20^{\circ} \mathrm{C}$ o máximo de ovos foi de 29 (Tabela 3). Glycaspis brimblecombei teve as médias de $31,2\left(22^{\circ} \mathrm{C}\right)$ e $31,7\left(26^{\circ} \mathrm{C}\right)$, mas, embora 
seja em outras temperaturas, observa-se que em $25^{\circ} \mathrm{C}$ as fêmeas de Blastopsylla occidentalis ovipositaram próximo à média encontrada em $26^{\circ} \mathrm{C}$ por Firmino (2004). Para Ctenarytaina eucalypti, as fêmeas podem ovipositar de 20 a 100 ovos, em condições naturais (QUEIROZ; BURCKHARDT; MAJER, 2012).

Tabela 3 - Número de fêmeas observadas, total de ovos $F_{1}$, média e variação de (ovos por dia \pm EP), número e taxa de eclosão (\%) de imaturos e duração média (X - \pm EP) dos períodos de incubação e intervalos de variação (dias) de imaturos $F_{1}$ de populações de Blastopsylla occidentalis Taylor, 1985 (Hemiptera: Aphalaridae), em cinco temperaturas em Sinop - MT, Brasil

\begin{tabular}{|c|c|c|c|c|c|c|}
\hline $\begin{array}{c}\text { Temperatura } \\
\left({ }^{\circ} \mathrm{C}\right)\end{array}$ & $\begin{array}{c}\text { Fêmeas } \\
\text { observadas }\end{array}$ & $\begin{array}{c}\text { Total } \\
\text { de } \\
\text { ovos } \\
F_{1} \\
\end{array}$ & $\begin{array}{c}\text { Média de } \\
\text { ovos } \\
F_{1} \pm E P \\
\text { (dias) }\end{array}$ & $\begin{array}{c}\text { Eclosão } \\
\text { de } \\
\text { imaturos } \\
F_{1}(\%) \\
\end{array}$ & $\begin{array}{l}\text { Imaturos } \\
\text { observados }\end{array}$ & $\begin{array}{c}\text { Período } \\
\text { incubação } \\
\text { Média } \pm \text { EP } \\
\text { (dias) }\end{array}$ \\
\hline & & & $19,04 \pm 0,91$ & & & $6,70 \pm 0,04$ \\
\hline \multirow[t]{2}{*}{20} & 38 & 476 & $\begin{array}{c}b \\
(11-29)\end{array}$ & 52 & 250 & $\begin{array}{c}b \\
(6-8)\end{array}$ \\
\hline & & & $26,40 \pm 1,33$ & & & $5,59 \pm 0,30$ \\
\hline 25 & 66 & 660 & $\begin{array}{c}a \\
(14-38)\end{array}$ & 85 & 566 & $\begin{array}{c}a \\
(5-7)\end{array}$ \\
\hline $30 *$ & 32 & - & - & - & - & - \\
\hline
\end{tabular}

Fonte: Autores (2020)

Em que: * não houve posturas. Médias seguidas pela mesma letra na coluna, não diferem significativamente entre si pelo teste de Scott-Knott a 5\% de probabilidade.

Observou-se que não houve diferença na duração média do período incubação da geração $F_{1}$ quando comparadas com a duração média de suas progenitoras, dessa maneira as fêmeas em $20^{\circ} \mathrm{C}$ tiveram a média de 6,97 enquanto para $F_{1}$ a média foi 6,70 dias, e em $25^{\circ} \mathrm{C}$, as médias foram de 5,80 dias para as fêmeas progenitoras e de 5,59 para imaturos da primeira geração. Ressalta-se que, para a primeira geração, ambas as temperaturas mantiveram a menor duração para eclosão que suas progenitoras, logo os imaturos eclodiram no mínimo de $6\left(20^{\circ} \mathrm{C}\right)$ e 5 dias $\left(25^{\circ} \mathrm{C}\right)$, porém houve redução de 1 dia na duração máxima para eclosão em $25^{\circ} \mathrm{C}$. 
Os resultados aqui apresentados corroboram a literatura que relacionam principalmente a temperatura $25^{\circ} \mathrm{C}$ para o melhor desenvolvimento e reprodução de insetos. Espera-se que esses resultados sirvam para conduzir estudos para a aplicação de técnicas de manejo para eventual controle da espécie.

\section{CONCLUSÃO}

A faixa de temperatura ótima para o desenvolvimento e reprodução de Blastopsylla occidentalis foi entre 20 e $25^{\circ} \mathrm{C}$, sendo que $25^{\circ} \mathrm{C}$ foi a temperatura em que o inseto apresentou menor mortalidade em todas as fases, menor tempo de desenvolvimento e maior número médio de ovos/dias. Em $30^{\circ} \mathrm{C}$, embora o inseto se desenvolva até a fase adulta, as fêmeas não ovipositaram e em $15^{\circ} \mathrm{C}$ e $35^{\circ} \mathrm{C}$ os insetos não completaram seu desenvolvimento.

Recomenda-se para a criação de Blastopsylla occidentalis, em laboratório, temperaturas entre 20 e $25^{\circ} \mathrm{C}$, as quais o inseto tem o seu melhor desenvolvimento desde o período incubação até a reprodução. O que pode auxiliar em futuros estudos com a espécie, inclusive para introdução de técnicas de manejo com predadores.

\section{AGRADECIMENTOS}

A primeira autora agradece o apoio da Coordenação de Aperfeiçoamento de Pessoal de NívelSuperior-Brasil(CAPES)-Código deFinanciamento 88882.459209/201901, pela concessão de bolsa de estudo de mestrado. À Embrapa Florestas através do Projeto $n^{\circ}$ 03.13.12.008.00.00. À Paula Pucci e Jaqueline Cremonese pela colaboração em testes de criação do psilídeo em laboratório na Embrapa Florestas. À Flora Sinop e a Fazenda Cunhatay pela colaboração.

T.B.S.K., D.L.Q. e M.R.B.: desenvolveram o projeto de pesquisa; T.B.S.K.: organizou e realizou o experimento; T.B.S.K e E.A.A.: analisaram os dados e realizaram os testes estatísticos; T.B.S.K.: escreveu o artigo; M.R.B. e D.L.Q.: orientaram a pesquisa; M.R.B., D.L.Q. e D.B.: revisaram para submissão da publicação no periódico. 


\section{REFERÊNCIAS}

BARBOSA, L. R. et al. Biological parameters, life table and thermal requirements of Thaumastocoris peregrinus (Heteroptera: Thaumastocoridae) at different temperatures. Scientific Report, [s. I.], v. 9, n. 1, p. 1-8, jul. 2019.

BURCKHARDT, D.; ELGUETA, M. Blastopsylla occidentalis Taylor (Hemiptera: Psyllidae), a new introduced eucalypt pest in Chile. Revista Chilena de Entomología, Santiago de Chile, v. 26, p. 57-61, 2000.

BURCKHARDT, D.; OUVRARD, D. A revised classification of the jumping plant-lice (Hemiptera: Psylloidea). Zootaxa, [s. I.], v. 3509, n. 1, p. 1-34, 2012.

COLINET, H. et al. Insects in fluctuating thermal environments. Annual Review of Entomology, Stanford, v. 60, p. 123-140, out. 2015.

FOOD AND AGRICULTURE ORGANIZATION OF THE UNITED NATIONS. Global Forest Resources Assessment 2015. Rome, 2015. Disponível em: http://www.fao.org/3/a-i4808e.pdf. Acesso em: 11 jan. 2020. (FAO Forestry Paper, n. 1).

FERREIRA, D. F. Sisvar: a computer statistical analysis system. Ciência e Agrotecnologia, Lavras, v. 35, n. 6, p. 1039-1042, nov./dez. 2011.

FIRMINO, D. C. Biologia do psilídeo-de-concha Glycaspis brimblecombei Moore (Hemiptera: Psyllidae) em diferentes espécies de eucalipto e em Eucalyptus camaldulensis sob diferentes temperaturas. 2004. Dissertação (Mestrado em Agronomia) - Faculdade de Ciências Agronômicas, Universidade Estadual Paulista, São Paulo, 2004. Disponível em: https:// www2.ipef.br/servicos/teses/arquivos/firmino,dc.pdf. Acesso em: 11 jan. 2020.

FONSECA, M. G. et al. How will Mahanarva spectabilis (Hemiptera: Cercopidae) Respond to Global Warming? Journal of Insect Science, [s. I.], v.16, n. 1, p. 1-6, mar. 2016.

HALL, D. G.; WENNINGER, E. J.; HENTZ, M. G. Temperature studies with the Asian citrus psyllid, Diaphorina citri: cold hardiness and temperature thresholds for oviposition. Journal of Insect Science, [s. I.], v. 11, n. 83, p. 1-15, jan. 2011.

HODKINSON, I. D. Life cycle variation and adaptation in jumping plant lice (Insecta: Hemiptera: Psylloidea): a global synthesis. Journal of natural History, London, v. 43, n. 1-2, p. 65-179, jan. 2009.

INDÚSTRIA BRASILEIRA DE ÁRVORES. Relatório 2019. São Paulo, 2019. Disponível em: https:// www.iba.org/datafiles/publicacoes/relatorios/iba-relatorioanual2019.pdf. Acesso em: 05 out. 2019.

LAWS, A. N.; BELOVSKY, G. E. How will species respond to climate change? Examining the effects of temperature and population density on an herbivorous insect. Environmental Entomology, College Park, v. 39, n. 2, p. 312-319, abr. 2010.

LONGUE-JÚNIOR, D.; COLODETTE, J. L. Importância e versatilidade da madeira de eucalipto para a indústria de base florestal. Pesquisa Florestal Brasileira, Colombo, v. 33, n. 76, p. 429438, out./dez. 2013.

OLIVEIRA, A. S. et al. Effect of temperature on the interaction between Chrysoperla externa (Neuroptera: Chrysopidae) and Sipha flava (Hemiptera: Aphididae). European Journal of Entomology, Branisovska, v. 107, n. 2, p. 183-188, abr. 2010. 
QUEIROZ, D. L.; BURCKHARDT, D.; MAJER, J. Integrated pest management of eucalypt psyllids (Insecta, Hemiptera, Psylloidea). In: LARRAMENDY, M. L.; SOLONESKI, S. (ed.). Integrated pest management and pest control: current and future tactics. Rijeka: InTech, 2012. p. 385-412.

QUEIROZ, D. L. et al. Criação de Blastopsylla occidentalis(Hemiptera: Aphalaridae) psilídeo das ponteiras do eucalipto, em casa de vegetação. [S. I.]: EMBRAPA Florestas, 2020. (Comunicado Técnico, 442).

QUEIROZ, D. L. et al. Feeding and oviposition preferences of Ctenarytaina spatulata Taylor (Hemiptera: Psyllidae) on Eucalyptus spp and other Myrtaceae growing in Brazil. Revista Brasileira de Entomologia, São Paulo, v. 54, p. 149-153, mar. 2010.

QUEIROZ, D. L. et al. New country, Brazilian states and host records of the eucalypt shoot psyllid Blastopsylla occidentalis. Pesquisa Florestal brasileira, Colombo, v. 38, e201701533, jun. 2018.

QUEIROZ, D. L. et al. Pragas em viveiro de eucalipto. In: WENDLING, I.; DUTRA, L. F. (org.). Produção de mudas de eucalipto. 2ed. Brasília: EMBRAPA, 2017. v. 1. p. 126-183.

QUEIROZ, D. L. S.; ZANOL, K. M. R. Biologia de Ctenarytaina spatulata (Hemiptera, Psyllidae) em Eucalyptus grandis. Acta Biológica Paranaense, Curitiba, v. 35, n. 1-2, p. 47-62, 2006.

RODRIGUES, W. C. Fatores que influenciam no desenvolvimento dos insetos. Info Insetos, [s. l.], v. 1, n. 4, p. 1-4, 2004.

SALIBA, I. L. et al. First record of Glycaspis brimblecombei (Moore, 1964) and Blastopsylla occidentalis (Taylor, 1985) (Hemiptera, Aphalaridae) in eucalyptus plantations in State of Pará, Brazil. Entomological Communications, [s. I.], v. 1, ec01009, dez. 2019.

SANTA-CECÍLIA, L. V. C. et al. Effect of temperature on development and survival of the mealybug cochineal Pseudococcus longispinus (Targioni Tozzetti, 1867) (Hemiptera: Pseudococcidae) in coffee plants. Coffee Science, Lavras, v. 6, n. 2, p. 1-7, maio/ago. 2011.

SANTANA, D. L. Q.; BURCKHARDT, D. Introduced Eucalyptus psyllids in Brazil. Journal of Forestry Research, Colombo, v. 12, p. 337-344, 2007.

SCHOWALTER, T. D. Insect ecology: an ecosystem approach. 4th ed. Massachusetts: Academic Press, 2016.

SCHÜHLI, G. S. et al. A review of the introduced forest pests in Brazil. Pesquisa Agropecuária Brasileira, Brasília, v. 51, n. 5, p. 397-406, maio 2016.

SOUZA, A. S. B.; KIRST, F. D. Aspectos da bionomia e metodologia de criação de dípteros de interesse forense. In: GOMES, L (org.). Entomologia Forense: novas tendências e tecnologias nas ciências criminais. 1. ed. Rio de Janeiro: Technical Books, 2010. p. 169-182.

VAN LIEROP, P. E. et al. Global forest area disturbance from fire, insect pests, diseases and severe weather events. Forest Ecologic and Management, [s. I.], v. 352, p. 78-88, dez. 2015.

WILCKEN, C. F. et al. Psilídeo-de-concha-do-eucalipto, Glycaspis brimblecombei Moore. In: VILELA, E. F.; ZUCCHI, R. A. (org.). Pragas introduzidas no Brasil: insetos e ácaros. [S. I.: s. n.], 2015. p. 883-897. 


\section{Contribuição de Autoria}

\section{1 - Talita Benedcta Santos Künast}

Bióloga, Ma.

https://orcid.org/0000-0003-4198-1039•talitakunast.bio@gmail.com

Contribuição: Conceituação, Investigação, Análise Formal, Escrita - primeira redação, Visualização

\section{2 - Dalva Luiz de Queiroz}

Engenheira Florestal, Dra., Pesquisadora

https://orcid.org/0000-0003-3556-9910•dalva.queiroz@embrapa.br

Contribuição: Conceituação, Metodologia, Escrita - revisão e edição

\section{3 - Daniel Burckhardt}

Biólogo, Dr., Curador

https://orcid.org/0000-0001-8368-5268•daniel.burckhardt@bs.ch

Contribuição: Conceituação, Escrita - revisão e edição

\section{4 - Ednaldo Antônio de Andrade}

Engenheiro Agrícola, Dr., Professor

https://orcid.org/0000-0001-6819-8904 • ednaldosnp@gmail.com

Contribuição: Análise Formal, Escrita - revisão e edição, Investigação

\section{5 - Marliton Rocha Barreto}

Biólogo, Dr., Professor

https://orcid.org/0000-0003-3793-8855•mrb.ufmt@gmail.com

Contribuição: Conceituação, Metodologia, Investigação, Escrita - revisão e edição

\section{Como citar este artigo}

Kunast, T. B.; Queiroz, D. L.; Burckhardt, D.; Andrade, E. A.; Barreto, M. R. Efeito da temperatura no desenvolvimento e na reprodução de Blastopsylla occidentalis (Hemiptera: Aphalaridae) em condições de laboratório. Ciência Florestal, Santa Maria, v. 31, n. 1, p. 440-455, 2021. DOI 10.5902/1980509843846. Disponível em: https://doi.org/10.5902/1980509843846. Acesso em: xx mês abreviado 202x. 